\title{
Clinical evaluation of vascular normalization induced by recombinant human endostatin in nasopharyngeal carcinoma via dynamic contrast-enhanced ultrasonography
}

This article was published in the following Dove Press journal:

OncoTargets and Therapy

\author{
Zhendong Yang' \\ Min Kang' \\ Shangyong Zhu ${ }^{2}$ \\ Jianyuan Huang ${ }^{2}$ \\ Xigui $\mathrm{Li}^{2}$ \\ Rensheng Wang' \\ 'Department of Radiation Oncology, \\ The First Affiliated Hospital \\ of Guangxi Medical University, \\ Guangxi Tumor Radiation Therapy \\ Clinical Medical Research Center, \\ Guangxi, People's Republic of China; \\ ${ }^{2}$ Department of Ultrasonography, \\ The First Affiliated Hospital of \\ Guangxi Medical University, Nanning, \\ Guangxi, People's Republic of China
}

Correspondence: Rensheng Wang Department of Radiation Oncology,

The First Affiliated Hospital of Guangxi Medical University, No 6, Shuangyong

Road, Nanning 53002I, Guangxi Zhuang Autonomous Region, People's Republic of China

Tel +86 I35 57386476

Email mdyangzhendong@I63.com
Background: The present study confirmed the presence and exact range of "vascular normalization window" induced by recombinant human endostatin (RHES) in patients with nasopharyngeal carcinoma (NPC) by analyzing the variation of dynamic contrast-enhanced ultrasonography (DCE-US) quantitative parameters. Also, the clinical application of DCE-US in the evaluation of vascular normalization was assessed.

Materials and methods: A total of 30 previously untreated patients with stage III-IVA NPC were enrolled in the present study and were randomly but equally divided into RHES (endostar [ES]) and normal saline (NS) groups. The patients in the ES group were administered RHES intravenously, while the patients in the NS group were administered normal saline daily for 5 days prior to intensity modulated radiotherapy coupled with concurrent chemotherapy. All patients underwent DCE-US on the day before the administration and on days 3 and 5 subsequently. The Audio Video Interleave of each DCE-US examination was analyzed quantitatively using the CHI-Q software. Several parameters were investigated, such as peak intensity (PI), time to peak (TTP), and mean transit time (MTT).

Results: The PI, TTP, and MTT differed significantly at the three time points in the ES group (all $P<0.001$ ) but not in the NS group (all $P>0.05$ ). In the ES group, PI increased and subsequently decreased, whereas TTP, as well as MTT, lessened initially and then increased within the 5 days after administration of RHES. The maximum value of PI and the minimum value of TTP, as well as MTT, occurred on day 3 (all $P<0.05$ ). Furthermore, the values of PI, TTP, and MTT were similar prior to the administration of RHES in both groups (all $P>0.05$ ). However, the PI of the ES group was significantly higher $(P<0.05)$, whereas the TTP and the MTT were significantly lower following administration of RHES (all $P<0.05$ ) compared with the corresponding parameters of the NS group.

Conclusion: DCE-US is a suitable method for the clinical evaluation of vascular normalization induced by antiangiogenic agents. The "vascular normalization window" induced by RHES occurs in patients with NPC, and the exact range is within about 5 days post-administration, which contributes towards optimizing the modality of RHES combined with radiotherapy and chemotherapy for NPC patients.

Keywords: evaluation, antiangiogenic, vascular normalization, endostatin, nasopharyngeal carcinoma, contrast-enhanced ultrasonography

\section{Introduction}

Nasopharyngeal carcinoma (NPC) exhibits a very unique pattern of geographical distribution, albeit with a low incidence worldwide. ${ }^{1}$ It is one of the most common 
cancers in the east and southeast regions of Asia, notably in Guangdong and Guangxi provinces of People's Republic of China. ${ }^{2}$ Intensity modulated radiotherapy (IMRT) combined with concurrent chemotherapy remains the primary and most effective treatment for NPC. It contributes to a prominent efficacy with a 5-year overall survival rate of $80 \%-90 \%$ as compared to the conventional 2-dimensional radiotherapy. ${ }^{3,4}$ However, a subset of patients with locoregionally advanced NPC continues to show poor prognosis, resulting from distant metastasis and recurrence in the nasopharynx or and/or regional lymph nodes..$^{5}$ The combination of antiangiogenic therapy with chemoradiotherapy provides a promising strategy for the improvement of the prognosis of such patients. ${ }^{6}$ Recombinant human endostatin (RHES) under the brand name of Endostar (ES) is a type of antiangiogenic agent. It was approved by the State Food and Drug Administration of China in September 2005 for the treatment of non-smallcell lung cancer (NSCLC). ${ }^{7}$ Since then, accumulated clinical data and experience have demonstrated that RHES could be effectively combined with radiotherapy and/or chemotherapy for the treatment of several malignant tumors, such as gastric cancers, metastatic melanoma, ${ }^{8,9}$ and NPC. ${ }^{10}$

According to the famous theory of starving tumor therapy that was proposed by Folkman in 1971, ${ }^{11}$ antiangiogenic therapy destroys the tumor vasculature, thereby depriving the tumor of oxygen and nutrients, which in turn suppresses the tumor growth. This is the primary mechanism underlying the anti-tumor effect the majority of the antiangiogenic agents including RHES. Nevertheless, excessive inhibition of tumor angiogenesis will result in hypoxia and impede the delivery of chemotherapeutic drugs to the tumor. Consequently, the efficacy of radiotherapy and chemotherapy is antagonized to a certain degree. ${ }^{12,13}$ In order to resolve this paradox, Jain and Lin and Sessa proposed the theories of "vascular normalization" and "window" successively. ${ }^{14,15}$ They reported that following administration of the antiangiogenic agents, a unique "window" occurred, where irregular vessels inside the tumor were normalized. Thus, the tumor blood flow increased, providing a valuable yet transient opportunity for improved delivery of the oxygen and chemotherapeutic agents that may enhance the curative effect. Furthermore, the precise range of the "window" differs by the different classes of antiangiogenic drugs and the histological types of the tumor. ${ }^{16-19}$ Apparently, the confirmation of the exact range of the "vascular normalization window" is crucial and difficult to accomplish due to its transient nature and uncertainty. Although the "vascular normalization window" induced by RHES in NPC has been proven in animal experiments, ${ }^{20}$ only a limited number of studies have conducted the clinical evaluation of vascular normalization.

Dynamic contrast-enhanced ultrasonography (DCE-US) is an advancing technology that has been developed in recent years and provides a promising application prospect for the assessment of the antiangiogenic therapy in many malignant tumors, ${ }^{21}$ owing to the obvious attractions of being non-allergic, non-invasive, widely available, portable, cost effective, highly sensitive, and reproducible. Therefore, the present study confirmed the presence and exact range of "vascular normalization window" induced by RHES in NPC patients by analyzing the variation of DCE-US parameters. In addition, we also estimated the clinical application of DCE-US in the evaluation of vascular normalization.

\section{Materials and methods}

This study was conducted in accordance with the Declaration of Helsinki and approved by the Ethics Committee at the First Affiliated Hospital of Guangxi Medical University. Written informed consent was obtained from all patients.

\section{Patients}

A total of 30 patients with stage III-IVA NPC who were diagnosed at the First Affiliated Hospital of Guangxi Medical University from July 2017 to July 2018 were enrolled in the present study. All patients were diagnosed by histopathological examinations and randomly divided into the ES group $(n=15)$ and the normal saline (NS) group $(n=15)$. The ES group included nine males and six females, aged between 28 and 70 years (median 41 years). The height of the patients in the ES group ranged from 150 to $172 \mathrm{~cm}$ (median $165 \mathrm{~cm}$ ) and the weight ranged from 48 to $77 \mathrm{~kg}$ (median $67 \mathrm{~kg}$ ). Among the patients in the ES group, one case was classified as WHO pathology type IIa, while the other 14 cases were WHO IIb. In addition, eight out of the 15 cases in the ES group had stage III NPC, whereas seven were with stage IV NPC. The NS group included eleven males and four females, aged between 28 and 68 years (median 49 years). The height of the patients in the NS group ranged from 152 to $172 \mathrm{~cm}$ (median $162 \mathrm{~cm}$ ) and the weight ranged from 42 to $73 \mathrm{~kg}$ (median $59 \mathrm{~kg}$ ). The 15 patients in the NS group were classified as WHO IIb. Moreover, five out of the 15 patients in the NS group exhibited stage III NPC, while the remaining ten were of stage IV NPC.

The inclusion criteria were as follows: histopathological diagnosis of NPC; no distant metastasis by auxiliary examination; initial diagnosis of NPC without previous radiotherapy or chemotherapy; stage III-IVA NPC according to 
the eighth edition of UICC/AJCC staging system for NPC; presence of NPC foci that were measurable according to the Response Evaluation Criteria in Solid Tumors standards; serum creatinine 1.25-fold of upper limit of normal (ULN) or creatinine clearance rate $\geq 60 \mathrm{~mL} / \mathrm{min}$; serum bilirubin 1.5-fold of ULN, aspartate amino transferase and alanine transaminase 2.5-fold of ULN, and alkaline phosphatase 5-fold of ULN; serum hemoglobin $\geq 10 \mathrm{gm} / \mathrm{dL}$, platelet count $\geq 100,000 / \mathrm{mL}$, absolute neutrophil count $\geq 1,500 / \mathrm{mL}$; Karnofsky scores $\geq 70$. The exclusion criteria were as follows: age $<18$ years or $>70$ years; allergic to SonoVue (Bracco, Milan, Italy); hemorrhagic tendency; cardiovascular right to left shunt; severe cardiac arrhythmias; myocardial infarction; severe pulmonary hypertension; active or recent gastrointestinal bleeding; diabetes mellitus with severe organ damage; severe end-organ damage; pregnancy or lactation; mental disorder; drug abuse or alcohol addiction.

\section{Administration methods}

Patients in the ES groups were administered RHES (solubilized in $250 \mathrm{~mL}$ of $0.9 \%$ normal saline) intravenously at a dosage of $7.5 \mathrm{mg} / \mathrm{m}^{2} /$ day for 5 days prior to IMRT coupled with concurrent chemotherapy. The patients in the NS group were administered $250 \mathrm{~mL}$ of $0.9 \%$ normal saline intravenously daily for 5 days prior to IMRT coupled with concurrent chemotherapy.

\section{DCE-US examination and parametric CEUS analysis}

All patients underwent DCE-US examinations on the day before the administration of RHES or normal saline and on days 3 and 5 subsequently. The patients were required to fast for a minimum of 8 hours prior to the initiation of the examinations. All the ultrasound (US) investigations were performed using the Aplio 500 ultrasound system (Toshiba, Tokyo, Japan) equipped with pulse subtraction contrast harmonic imaging and a $2.5-5.0 \mathrm{MHz}$ convex-array transducer. SonoVue was selected as the intravenous contrast medium. For the accurate positioning of the nasopharyngeal tumor, a specific body position of the patients was required: the patients were placed in the supine position with the neck biased towards the opposite side and slightly tilted back. The transducer was placed between the mastoid and mandible ramus aspect of the neck, and the nasopharynx and parapharyngeal space were examined in transverse, longitudinal, and oblique planes. The parotid gland and cervical vessels could be used as acoustic windows. ${ }^{22}$ Following positioning of the tumor, a bottle of SonoVue was dissolved in $5 \mathrm{~mL}$ of saline and a $2.4 \mathrm{~mL}$ bolus was injected into the superficial elbow vein of each patient at the rate of $1 \mathrm{~mL} / \mathrm{sec}$, followed by a $5.0 \mathrm{~mL}$ saline flush. Simultaneously, a video comprising the DCE-US imaging was recorded for at least 60 seconds. The video was stored as a digital archive (Audio Video Interleave, AVI) in the hard disc for post-parametric analysis. ${ }^{23}$

The AVI of each DCE-US examination was analyzed quantitatively through CHI-Q software (Toshiba) in order to obtain a series of parameters. The detailed protocol was as follows: initially, the nasopharyngeal tumor was set as the region of interest (ROI); subsequently, the ROI was analyzed to obtain the time/intensity curve (TIC) and several parameters, including peak intensity (PI), time to peak (TTP), and mean transit time (MTT). PI was defined as the maximum value of the signal intensity, which was significantly correlated with the quantity of intravascular microbubble, reflecting objectively the blood volume inside the tumor microvessel. TTP was defined as the time from base intensity to maximum intensity, and MTT was defined as the mean time required by the bubbles to pass through the tumor. TTP and MTT both indicated the blood flow velocity inside the tumor microvessel. ${ }^{23}$ Each AVI was analyzed three times, and the average value for each parameter was calculated. The average value was based on the final data obtained for the specific time point of the DCE-US examination. In order to maintain the quality and consistency of the imaging, the DCE-US examination and quantitative analysis for all 30 patients were performed by the same US sonographer. The investigator had more than 5 years of experience in the application of CEUS on the same equipment.

\section{Statistical analysis}

The data were analyzed by using SPSS 23.0 (IBM Corporation, Armonk, NY, USA). The measurement data were expressed as mean $\pm \mathrm{SD}$. The $t$-test was used to compare the mean between the different groups. The repeated measure analysis of variance and the Bonferroni $t$-test were used to analyze the continuous variables of the same group. Statistical significance was set at $P<0.05$.

\section{Results}

The baseline characteristics of the patients including gender, ages, height, weight, pathology types, and staging between the two groups were similar (Table 1). Color Doppler ultrasonography imaging of NPC is shown in Figure 1. The DCE-US imaging and TIC varied significantly at different time points in the patients of the ES group (Figure 2), although they were the same in the NS group (Figure 3). 
Table I Baseline characteristics of patients

\begin{tabular}{|c|c|c|c|}
\hline \multirow[t]{2}{*}{ Characteristics } & \multicolumn{2}{|l|}{ Groups } & \multirow[t]{2}{*}{$P$-value } \\
\hline & ES $(n=15)$ & NS $(n=15)$ & \\
\hline Gender (cases) & & & 0.700 \\
\hline Male & 9 & 11 & \\
\hline Female & 6 & 4 & \\
\hline Age (years) & & & 0.214 \\
\hline Median & 41 & 49 & \\
\hline Range & $28-70$ & $28-68$ & \\
\hline Height (cm) & & & 0.783 \\
\hline Median & 165 & 162 & \\
\hline Range & $150-172$ & $152-172$ & \\
\hline Weight (kg) & & & 0.199 \\
\hline Median & 67 & 59 & \\
\hline Range & $48-77$ & $42-73$ & \\
\hline Pathology types ${ }^{\mathrm{a}}$ (cases) & & & 1.000 \\
\hline WHO I & 0 & 0 & \\
\hline WHO Ila & 1 & 0 & \\
\hline WHO Ilb & 14 & 15 & \\
\hline WHO III & 0 & 0 & \\
\hline T staging ${ }^{\mathrm{b}}$ (cases) & & & 0.715 \\
\hline TI & 0 & 0 & \\
\hline $\mathrm{T} 2$ & 0 & 0 & \\
\hline T3 & 9 & 7 & \\
\hline $\mathrm{T} 4$ & 6 & 8 & \\
\hline $\mathrm{N}$ staging ${ }^{\mathrm{b}}$ (cases) & & & 0.609 \\
\hline No & 2 & 2 & \\
\hline $\mathrm{NI}$ & 5 & 2 & \\
\hline N2 & 7 & 9 & \\
\hline N3 & 1 & 2 & \\
\hline Clinical staging (cases) & & & 0.462 \\
\hline III & 8 & 5 & \\
\hline IVA & 7 & 10 & \\
\hline
\end{tabular}

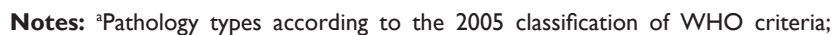
'T staging according to the eighth edition of UICC/AJCC staging system for NPC; $P$-values were calculated by the Fisher's exact test or chi-squared test. Abbreviations: ES, Endostar; NS, normal saline.

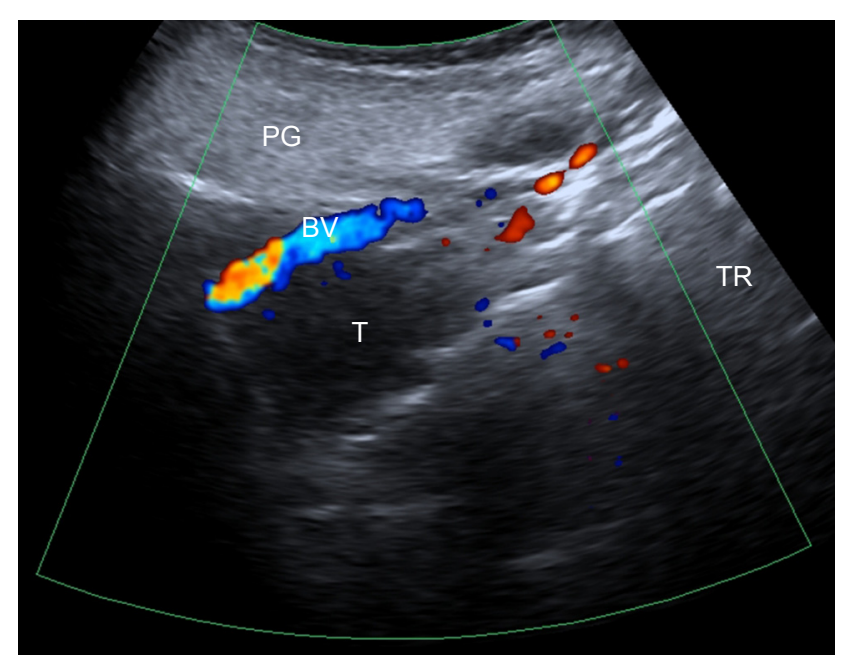

Figure I Color Doppler ultrasonography imaging of NPC in a 60-year-old female. Notes: A large focal mass was detected in the nasopharynx; the parotid gland and cervical blood vessels were used as acoustic windows.

Abbreviations: BV, cervical blood vessel; NPC, nasopharyngeal carcinoma; PG, parotid gland; T, tumor; TR, tongue root.
The PI values of the patients in the ES group on the day before (PIpre) and days 3 (PId3) and 5 (PId5) following administration of RHES were $-47.31 \pm 3.81(\mathrm{~dB}),-39.22 \pm$ $3.45(\mathrm{~dB})$, and $-43.59 \pm 3.86(\mathrm{~dB})$, respectively. In the NS group, the values of PIpre, PId3, and PId5 were $-48.08 \pm$ $3.11(\mathrm{~dB}),-47.73 \pm 2.83(\mathrm{~dB})$, and $-48.47 \pm 2.94(\mathrm{~dB})$, respectively. The PI values of the subjects who were examined in the three time points of the ES group differed significantly $(P<0.001)$, whereas the comparison of the corresponding values of the parameters in the NS group did not exhibit significant differences $(P=0.330)$. Furthermore, a comparison between the ES and NS groups did not reveal any significant differences in the PIpre $(P=0.551)$, while the values of PId3 and PId5 of the ES group were significantly higher than those of the NS group (all $P<0.05$ ) (Table 2). The PI values of the patients in the ES group initially increased and subsequently decreased within the 5 days following administration of RHES. The maximum value was observed on day 3 (Figure 4).

The TTP values of the patients in the ES group on the day before (TTPpre) and on days 3 (TTPd3) and 5 (TTPd5) fol-

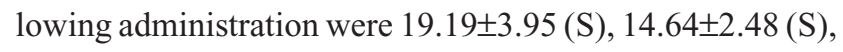
and $16.34 \pm 2.29(\mathrm{~S})$, respectively. The TTPpre, TTPd3, and TTPd5 of the NS group were 19.19 \pm 3.92 (S), 19.12 \pm 3.18 (S), and 19.59 $\pm 3.61(\mathrm{~S})$, respectively. Thus, a significant difference was detected in the TTP values of the ES group among the three different time points $(P<0.001)$, while no significant difference was observed in the NS group $(P=0.556)$. The TTPpre values of the ES and NS groups were similar $(P=1.000)$, while the TTPd3 and TTPd5 values were significantly lower in the ES group as compared to those of the NS group (all $P<0.05$ ) (Table 3). Moreover, the MTT values of the patients in the ES group on the day prior to RHES administration (MTTpre) and on days 3 (MTTd3) and 5 (MTTd5) following administration were $29.41 \pm 4.53$ (S), $21.85 \pm 5.40(\mathrm{~S})$, and $24.96 \pm 4.33(\mathrm{~S})$, respectively. The values of the MTTpre, MTTd3, and MTTd5 of the NS group were $28.94 \pm 5.03$ (S), 28.41 $\pm 4.66(\mathrm{~S})$, and 29.44 \pm 3.89 (S), respectively. Thus, a significant difference was observed in the ES group among the three time points $(P<0.001)$, whereas none was detected in the NS group $(P=0.284)$. The MTTpre of the ES group differed significantly compared with that of the NS group $(P=0.791)$. Nevertheless, the values of MTTd3 and MTTd5 were significantly lower (all $P<0.05$ ) (Table 4 ). Therefore, the TTP and MTT values of the patients in the ES group initially reduced and subsequently extended within 5 days following the administration of RHES. The minimum value was noted on day 3 (Figure 4). 


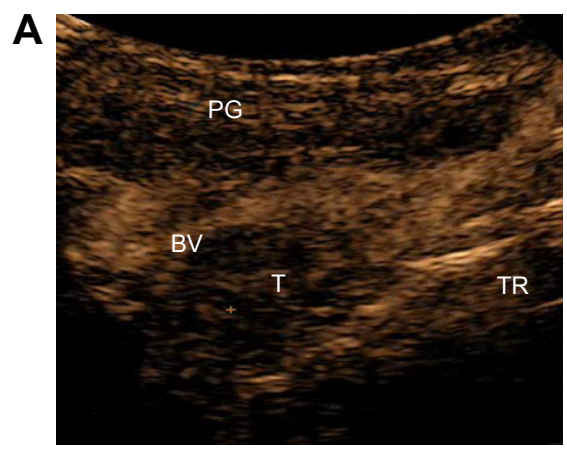

D

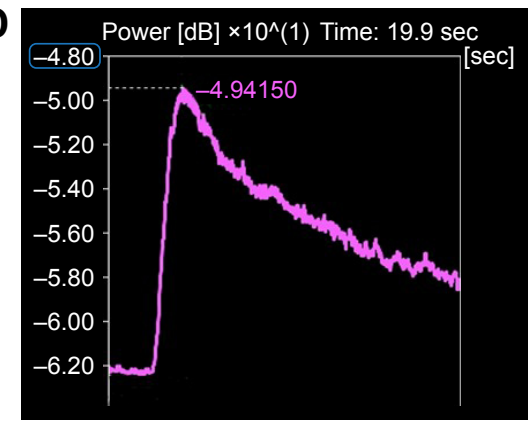

B

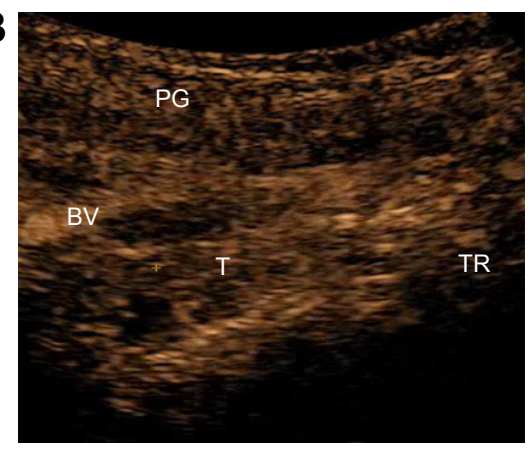

E

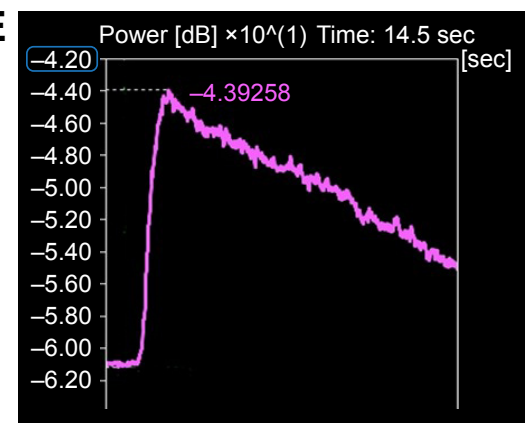

C

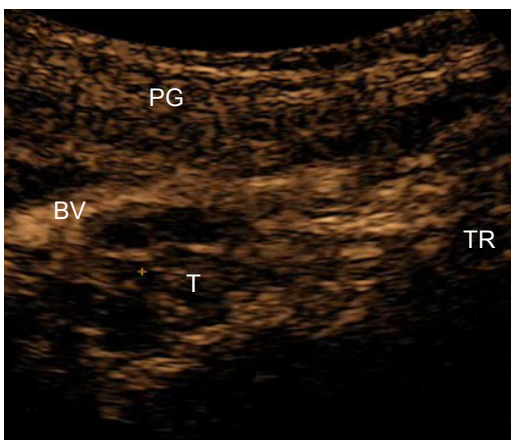

$\mathbf{F}$

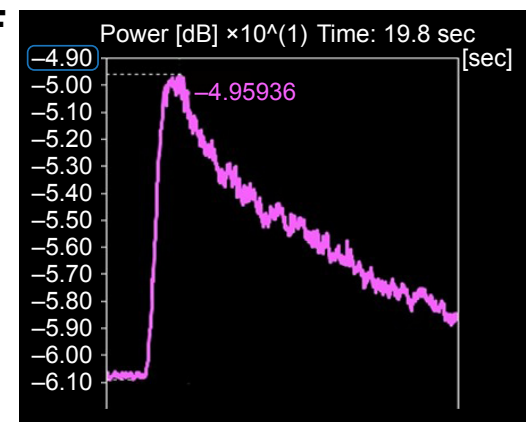

Figure 2 DCE-US imaging and TIC of NPC in a 60-year-old female of the ES group at different time points after administration of RHES.

Notes: (A-C) DCE-US imaging of NPC in a 60-year-old female on the day before RHES administration and on days 3 and 5 after administration of RHES, respectively. (D-F) TIC in the corresponding imaging of A-C, respectively. PI initially increased and subsequently decreased, whereas the opposite pattern was noted for TTP. These changes were noticeable within 5 days following administration of RHES, whereas the maximum and minimum values of PI and TTP, respectively, occurred on day 3 , suggesting the presence of the "vascular normalization window".

Abbreviations: BV, cervical blood vessel; DCE-US, dynamic contrast-enhanced ultrasonography; ES, Endostar; NPC, nasopharyngeal carcinoma; PG, parotid gland; PI, peak intensity; RHES, recombinant human endostatin; T, tumor; TIC, time/intensity curve; TR, tongue root; TTP, time to peak.

\section{A}

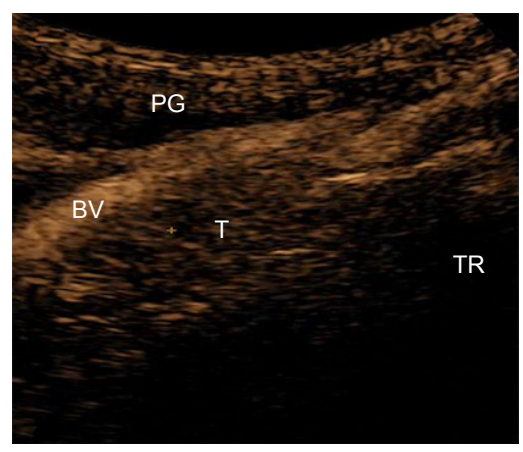

D

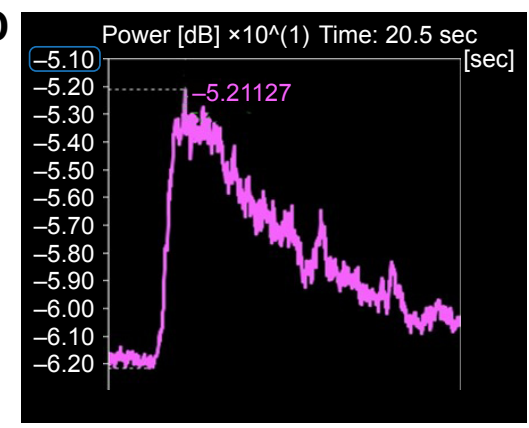

B

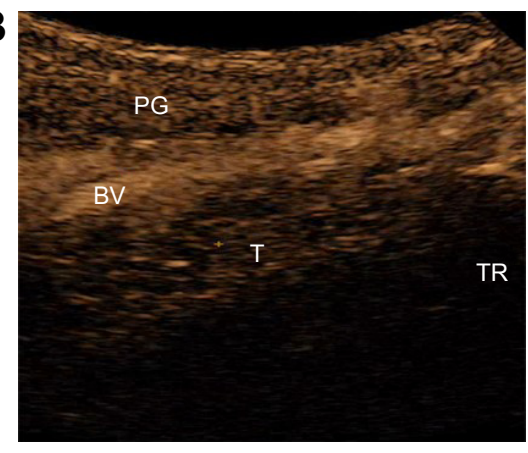

E

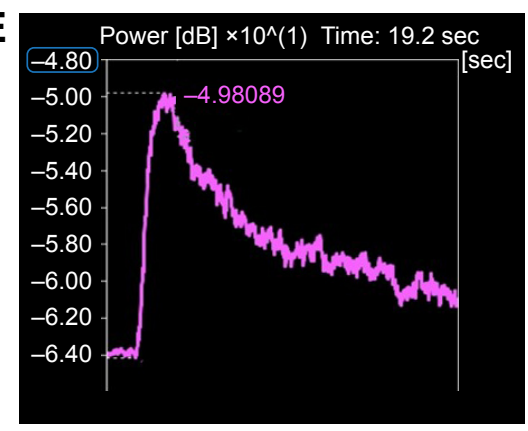

C

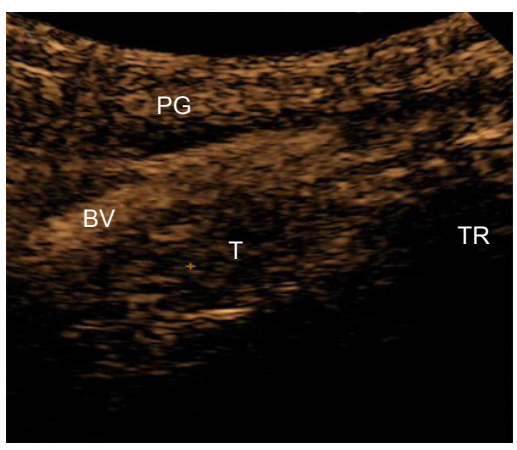

F

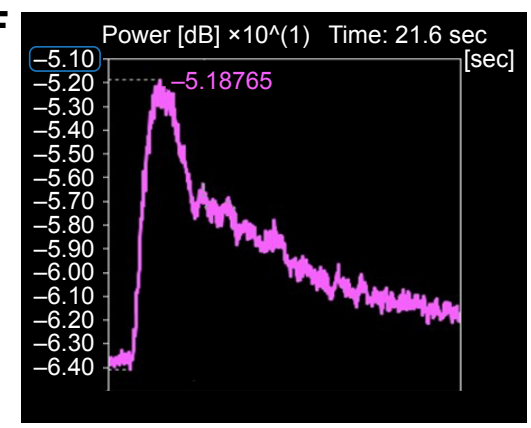

Figure 3 DCE-US imaging and TIC of NPC in a 56-year-old male of the NS group at different time points following administration of normal saline.

Notes: (A-C) DCE-US imaging of NPC in a 56-year-old male on the day before administration of NS and on days 3 and 5 after administration of NS, respectively. (D-F) TIC in the corresponding imaging of A-C, respectively. PI and TTP almost equally distributed among the three time points following administration of NS.

Abbreviations: BV, cervical blood vessel; DCE-US, dynamic contrast-enhanced ultrasonography; NPC, nasopharyngeal carcinoma; NS, normal saline; PG, parotid gland;

$\mathrm{PI}$, peak intensity; T, tumor; TIC, time/intensity curve; TR, tongue root; TTP, time to peak. 
Table 2 Variation of $\mathrm{PI}(\mathrm{dB})$ before and after administration

\begin{tabular}{|c|c|c|c|c|c|}
\hline \multirow[t]{2}{*}{ Groups } & \multicolumn{3}{|c|}{ PI (dB) before and after administration } & \multirow[t]{2}{*}{$F^{a}$} & \multirow[t]{2}{*}{$P$-value } \\
\hline & Pre & d3 & d5 & & \\
\hline$E S(n=15)$, mean $\pm S D$ & $-47.31 \pm 3.81$ & $-39.22 \pm 3.45$ & $-43.59 \pm 3.86$ & 63.808 & 0.000 \\
\hline$N S(n=15)$, mean $\pm S D$ & $-48.08 \pm 3.11$ & $-47.73 \pm 2.83$ & $-48.47 \pm 2.94$ & 1.152 & 0.330 \\
\hline$t^{\mathrm{b}}$ & 0.604 & 7.385 & 3.894 & & \\
\hline$P$-value ${ }^{b}$ & 0.551 & 0.000 & 0.001 & & \\
\hline
\end{tabular}

Notes: aBy repeated measure analysis of variance; by independent-samples $t$-test.

Abbreviations: $\mathrm{d} 3$, day 3 after administration; d5, day 5 after administration; ES, Endostar; NS, normal saline; PI, peak intensity; Pre, the day before administration.

No side effect related with RHES or DCE-US, such as allergy, headache, arrhythmia, nausea, and fever, was observed in all 30 patients.

\section{Discussion}

RHES is an effective antiangiogenic agent that enhances the treatment efficacy for advanced NPC according to previous studies. ${ }^{24-26}$ However, hypoxia and less amount of drug deliver, as a result of antiangiogenesis, may limit the synergistic effect of RHES combined with radiotherapy and chemotherapy. Based on the studies by Jain ${ }^{14}$ and Lin and Sessa, ${ }^{15}$ this paradox can be resolved by administering the radiotherapy and chemotherapy in a specific yet transient time frame, which was defined as "vascular normalization window". In this unique "window", the improved blood perfusion, more oxygen and more chemotherapeutic agents were delivered to the tumor. Obviously, the clinical evaluation of the exact range of vascular normalization window to optimize the synergistic effect of RHES coupled with radiotherapy and chemotherapy is critical and challenging.

The gold standards for the evaluation of angiogenesis and vascular normalization remain the microvessel density (MVD), vascularity, basement membrane, and pericyte coverage. ${ }^{27}$ However, their clinical applications are difficult due to the invasive nature and dependency on qualified biopsy. The imageological examinations, such as positron emission computed tomography, DCE-MRI, and CT perfusion imaging (CTPI), were used to assess the vascular normalization in animal experiments and clinical studies. ${ }^{28-30}$ Nevertheless, these examinations present disadvantages,

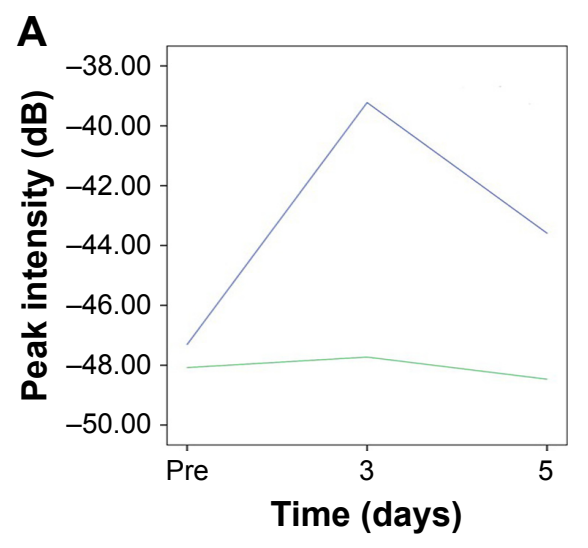

B

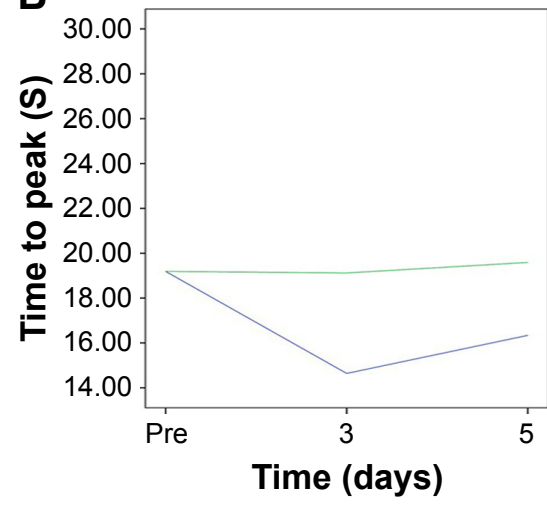

- ES group - NS group

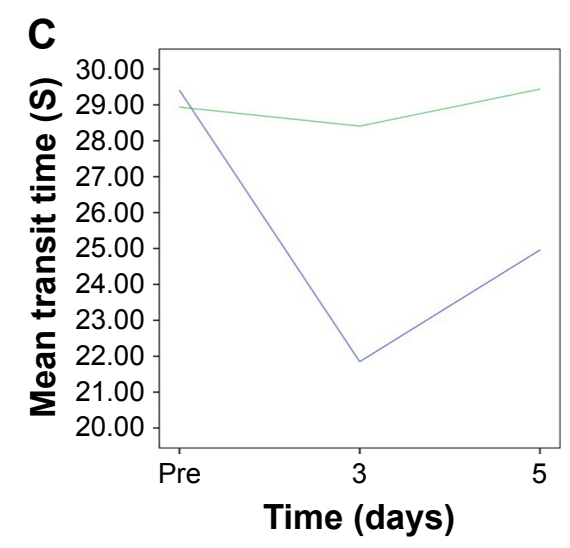

Figure 4 Line chart of DCE-US parameters in NPC patients.

Notes: (A) PI in the ES group initially increased and subsequently decreased within the 5 days following administration of RHES. The maximum value occurred on day 3 $(P<0.00 \mathrm{I})$, while it remained stable in the NS group $(P=0.330)$. The $\mathrm{PI}$ values were similar in the two groups prior to RHES administration $(P=0.55 \mathrm{I})$. However, the $\mathrm{PI}$ of the ES group was significantly higher following administration of RHES (all $P<0.05$ ). (B) The TTP values in the ES group initially reduced and subsequently increased within the 5 days following administration of RHES. The minimum value appeared on day $3(P<0.00 \mathrm{I})$, and it did not exhibit a significant change in the NS group $(P=0.556)$. The TTP values were similar in the two groups before administration of RHES $(P=1.000)$, whereas the TTP value in the ES group initially increased and subsequently decreased within the 5 days following administration of RHES (all $P<0.05$ ). (C) The MTT value in the ES group initially increased and subsequently decreased within the 5 days following administration of RHES. The minimum value appeared on day $3(P<0.00 \mathrm{I})$, while it exhibited no significant change in the NS group $(P=0.284)$. The MTT values were similar in the two groups prior to administration of RHES $(P=0.791)$. However, the MTT value of the ES group was significantly lower following administration of RHES (all $P<0.05)$. These significant wave-like variations of the DCE-US parameters in the patients of the ES group indicated strongly the presence of the "vascular normalization window".

Abbreviations: DCE-US, dynamic contrast-enhanced ultrasonography; ES, Endostar; MTT, mean transit time; NPC, nasopharyngeal carcinoma; NS, normal saline; PI, peak intensity; RHES, recombinant human endostatin; TTP, time to peak. 
Table 3 Variation of TTP (S) before and after administration

\begin{tabular}{|c|c|c|c|c|c|}
\hline \multirow[t]{2}{*}{ Groups } & \multicolumn{3}{|c|}{ TTP (S) before and after administration } & \multirow[t]{2}{*}{$F^{a}$} & \multirow[t]{2}{*}{$P$-value } \\
\hline & Pre & d3 & d5 & & \\
\hline$E S(n=15)$, mean $\pm S D$ & $19.19 \pm 3.95$ & $14.64 \pm 2.48$ & $16.34 \pm 2.29$ & 22.338 & 0.000 \\
\hline$N S(n=15)$, mean $\pm S D$ & $19.19 \pm 3.92$ & $19.12 \pm 3.18$ & $19.59 \pm 3.61$ & 0.599 & 0.556 \\
\hline$t^{\mathrm{b}}$ & 0.000 & -4.297 & -2.942 & & \\
\hline$P$-value ${ }^{b}$ & 1.000 & 0.000 & 0.006 & & \\
\hline
\end{tabular}

Notes: ${ }^{\mathrm{B}}$ By repeated measure analysis of variance; 'by independent-samples $t$-test.

Abbreviations: $\mathrm{d} 3$, day 3 after administration; d5, day 5 after administration; ES, Endostar; NS, normal saline; Pre, the day before administration; TTP, time to peak.

such as ionizing radiation, high cost, allergy to contrast agent, and poor reproducibility, which might limit their application in routine clinical practice.

As a milestone in the history of ultrasonic medicine, DCE-US technology possesses attractive advantages in evaluation of vascular normalization, such as allergen free, radiation free, noninvasiveness, cost-effectiveness, and optimal repeatability. ${ }^{31}$ Moreover, unlike iodinated and gadolinium contrast agents, the microbubble ultrasound contrast agents with a diameter of 3-5 $\mu \mathrm{m}$ are solely confined to the blood pool without interferences from the extravasations of the contrast agent. Thus, accurate measurements that are related to vascular normalization inside the tumor are acquired. ${ }^{32}$ DCE-US can record the distribution, concentration, and dissipation of microbubble and provide TIC in addition to several parameters (eg, PI, TTP, and MTT) via quantitative analysis of the AVI of DCE-US. These parameters can be categorized as predominantly hemodynamic or predominantly morphological, or a combination of the two according to the introduction of DCE-US formulated by the European Federation of Societies for Ultrasound in Medicine and Biology. ${ }^{23}$ PI was defined as the maximum value of the signal intensity that was significantly correlated with the quantity of intravascular microbubble, reflecting authentically of the blood volume inside the tumor microvessel. TTP was defined as the time from base intensity to maximum intensity, whereas MTT was defined as the mean time taken by the bubbles to pass through the tumor. TTP and MTT can reflect the blood flow velocity inside the tumor microvessel. In addition, MVD is one of the gold standards for the assessment of angiogenesis and vascular normalization. ${ }^{27}$ Mori et $\mathrm{al}^{133}$ reported that the parameters of CEUS, such as PI, was significantly correlated with MVD in breast cancer $(r=0.43)$. The correlation exhibited a significant difference $(P=0.0073)$. This conclusion was similar to the result $(r=0.267, P=0.017)$ based on a clinical study led by Li et al. ${ }^{34}$ Thus, the dynamic variation of the parameters of CEUS can reflect the progress of vascular normalization to a certain extent.

In the current study, the PI of the patients with NPC in the ES group increased initially and decreased subsequently within the 5-day period following RHES. The maximal values were observed on day 3 . It is interesting to note that the TTP of the patients in the ES group initially decreased and subsequently increased. The minimal values appeared on day 3. The variation of MTT was the same as that of TTP. Conversely, the parameters PI, TTP, and MTT, of the patients in the NS group remained almost unaltered within the 5 days following administration. Furthermore, the values of the patients in the ES group were similar to those of the NS group prior to the administration of the RHES. However, the PI value was significantly higher than the corresponding value of the NS group, whereas the TTP and MTT value were significantly lower than that of the NS group after administration. Wave-like variations were observed with regard to the DCE-US parameters following administration of RHES, which indicated the presence of the "vascular normalization

Table 4 Variation of MTT (S) before and after administration

\begin{tabular}{|c|c|c|c|c|c|}
\hline \multirow[t]{2}{*}{ Groups } & \multicolumn{3}{|c|}{ MTT (S) before and after administration } & \multirow[t]{2}{*}{$\mathbf{F}^{\mathbf{a}}$} & \multirow[t]{2}{*}{$P$-value } \\
\hline & Pre & d3 & d5 & & \\
\hline ES $(n=15)$, mean $\pm S D$ & $29.4 I \pm 4.53$ & $21.85 \pm 5.40$ & $24.96 \pm 4.33$ & 30.507 & 0.000 \\
\hline NS $(n=15)$, mean $\pm S D$ & $28.94 \pm 5.03$ & $28.4 I \pm 4.66$ & $29.44 \pm 3.89$ & 1.317 & 0.284 \\
\hline$t^{\mathrm{b}}$ & 0.267 & -3.563 & -2.978 & & \\
\hline$P$-value ${ }^{b}$ & 0.791 & 0.001 & 0.006 & & \\
\hline
\end{tabular}

Notes: ${ }^{a}$ By repeated measure analysis of variance; by independent-samples $t$-test.

Abbreviations: $\mathrm{d} 3$, day 3 after administration; d5, day 5 after administration; ES, Endostar; MTT, mean transit time; NS, normal saline; Pre, the day before administration. 
window" induced by RHES. The exact range of the window was approximately 5 days following administration of RHES. These results were similar to conclusions reported by Jiang et al, ${ }^{30}$ where the "vascular normalization window" induced by RHES was evaluated in patients with NSCLC by CTPI. A "time window" was demonstrated that corresponded to a week following administration of RHES. Li et al demonstrated in a A549 lung adenocarcinoma xenograft murine model that the "vascular normalization window" induced by RHES was evident from day 4 to day 10 following administration of RHES. ${ }^{19}$ Peng et $\mathrm{al}^{20}$ reported that the RHES could induce "vascular normalization window" in mice bearing CNE-2 and 5-8F human NPC xenografts, thereby causing an array of transient pathophysiological changes to tumor microvessels, such as the decline in tumor vascularity and in the hypoxic tumor cell fraction, and the increase of the basement membrane and the pericyte coverage associated with endothelial cells. In the present study, the regular variations of the DCE-US parameters in the NPC patients may relate to several pathophysiological mechanisms. In the early stage after administration of RHES, the inordinate tumor microenvironment is reconstructed, resulting in an increase in the basement membrane and the pericyte coverage. Concomitantly, the permeability of the microvessel was reduced, leading to increased blood perfusion, larger blood volume, and accelerated blood flow velocity. These pathological changes were reflected as enhancement of PI and shortening of TTP and MTT in the quantitative analysis of DCE-US. Subsequently, the antiangiogenic effects of RHES were initiated and the tumor microvessel was regressed, resulting in lower blood perfusion and reduced blood volume and blood flow velocity. These were accordingly reflected as a decrease in the PI value and an increase in the TTP and MTT values.

\section{Limitations and prospect}

Several limitations should be emphasized. First, the veracity of DCE-US relied on the experience of the operators and the quality of ultrasound equipment. Second, the number of enrolled patients was small in this present study. The statistical strength could have decreased owing to the abovementioned factors. Therefore, further research is needed. A further study with more patients and longer follow-up may provide more compelling evidence for the existence of "vascular normalization window" induced by RHES in NPC patients. Furthermore, the "vascular normalization window" of NPC patients may vary with different characteristics (such as age, sex, size of the tumor, weight and height). A further study with large samples may provide data to confirm the "vascular normalization window" and the optimal timing of performing chemoradiotherapy for each subgroups, thus to improve the therapeutic effect.

\section{Conclusion}

The present study suggests that DCE-US exhibits certain advantages, such as allergen free, radiation free, noninvasiveness, cost-effectiveness, and optimal reproducibility. Therefore, DCE-US is a suitable method for the clinical evaluation of vascular normalization induced by antiangiogenic agents. Furthermore, the results confirmed the presence of "vascular normalization window" induced by RHES in patients with NPC. The exact range was within 5 days following administration of RHES, which contributes toward optimizing the modality of RHES combined with radiotherapy and chemotherapy for NPC patients.

\section{Acknowledgment}

This work was supported by the Foundation of Sharing Project Based on Tumor Precise Radiotherapy (ZY18076006).

\section{Disclosure}

The authors report no conflicts of interest in this work.

\section{References}

1. Ferlay J, Soerjomataram I, Dikshit R, et al. Cancer incidence and mortality worldwide: sources, methods and major patterns in GLOBOCAN 2012. Int J Cancer. 2015;136(5):E359-E386.

2. Wei KR, Zheng RS, Zhang SW, Liang ZH, Li ZM, Chen WQ. Nasopharyngeal carcinoma incidence and mortality in China, 2013. Chin $J$ Cancer. 2017;36(1):90.

3. Zhang MX, Li J, Shen GP, et al. Intensity-modulated radiotherapy prolongs the survival of patients with nasopharyngeal carcinoma compared with conventional two-dimensional radiotherapy: A 10-year experience with a large cohort and long follow-up. Eur J Cancer. 2015; 51(17):2587-2595

4. Peng G, Wang T, Yang KY, et al. A prospective, randomized study comparing outcomes and toxicities of intensity-modulated radiotherapy vs. conventional two-dimensional radiotherapy for the treatment of nasopharyngeal carcinoma. Radiother Oncol. 2012;104(3):286-293.

5. Au KH, Ngan RKC, Ng AWY, et al. Treatment outcomes of nasopharyngeal carcinoma in modern era after intensity modulated radiotherapy (IMRT) in Hong Kong: A report of 3328 patients (HKNPCSG 1301 study). Oral Oncol. 2018;77:16-21.

6. Lee NY, Zhang Q, Pfister DG, et al. Addition of bevacizumab to standard chemoradiation for locoregionally advanced nasopharyngeal carcinoma (RTOG 0615): a phase 2 multi-institutional trial. Lancet Oncol. 2012;13(2):172-180.

7. Wang J, Sun Y, Liu Y, et al. Results of randomized, multicenter, doubleblind phase III trial of rh-endostatin (YH-16) in treatment of advanced non-small cell lung cancer patients. Zhongguo Fei Ai Za Zhi. 2005; 8(4):283-290. Chinese.

8. Xu R, Ma N, Wang F, et al. Results of a randomized and controlled clinical trial evaluating the efficacy and safety of combination therapy with Endostar and S-1 combined with oxaliplatin in advanced gastric cancer. Onco Targets Ther. 2013;6:925-929. 
9. Cui C, Mao L, Chi Z, et al. A phase II, randomized, double-blind, placebo-controlled multicenter trial of Endostar in patients with metastatic melanoma. Mol Ther. 2013;21(7):1456-1463.

10. YY L, Jin F. Clinical results of recombinant human endostatin combined with chemotherapy and radiation therapy for locally advanced nasopharyngeal carcinoma: a Phase 2 multi-institutional trial. Int $J$ Radiat Oncol Biol Phys. 2016;96(2):E378.

11. Folkman J. Tumor angiogenesis: therapeutic implications. $N$ Engl J Med. 1971;285(21):1182-1186.

12. Fenton BM, Paoni SF, Ding I. Effect of VEGF receptor-2 antibody on vascular function and oxygenation in spontaneous and transplanted tumors. Radiother Oncol. 2004;72(2):221-230.

13. Ma J, Pulfer S, Li S, Chu J, Reed K, Gallo JM. Pharmacodynamicmediated reduction of temozolomide tumor concentrations by the angiogenesis inhibitor TNP-470. Cancer Res. 2001;61(14):5491-5498.

14. Jain RK. Normalizing tumor vasculature with anti-angiogenic therapy: a new paradigm for combination therapy. Nat Med. 2001;7(9): 987-989.

15. Lin MI, Sessa WC. Antiangiogenic therapy: creating a unique "window" of opportunity. Cancer Cell. 2004;6(6):529-531.

16. Chen DR, Lin C, Wang YF. Window of opportunity: A new insight into sequential bevacizumab and paclitaxel in two cases of metastatic triple-negative breast cancer. Exp Ther Med. 2015;10(3):885-888.

17. Vangestel C, Van de Wiele C, Van Damme N, et al. (99)mTc-(CO)(3) His-annexin A5 micro-SPECT demonstrates increased cell death by irinotecan during the vascular normalization window caused by bevacizumab. J Nucl Med. 2011;52(11):1786-1794.

18. Batchelor TT, Sorensen AG, di Tomaso E, et al. AZD2171, a pan-VEGF receptor tyrosine kinase inhibitor, normalizes tumor vasculature and alleviates edema in glioblastoma patients. Cancer Cell. 2007;11(1): 83-95.

19. Li N, Zheng D, Wei X, Jin Z, Zhang C, Li K. Effects of recombinant human endostatin and its synergy with cisplatin on circulating endothelial cells and tumor vascular normalization in A549 xenograft murine model. J Cancer Res Clin Oncol. 2012;138(7):1131-1144.

20. Peng F, Xu Z, Wang J, et al. Recombinant human endostatin normalizes tumor vasculature and enhances radiation response in xenografted human nasopharyngeal carcinoma models. PLoS One. 2012;7(4): e34646.

21. Lassau N, Chapotot L, Benatsou B, et al. Standardization of dynamic contrast-enhanced ultrasound for the evaluation of antiangiogenic therapies: the French multicenter Support for Innovative and Expensive Techniques Study. Invest Radiol. 2012;47(12):711-716.

22. Gao Y, Liu JJ, Zhu SY, Yi X. The Diagnostic Accuracy of Ultrasonography versus Endoscopy for Primary Nasopharyngeal Carcinoma. PLoS One. 2014;9(6):e99679.
23. Dietrich CF, Averkiou MA, Correas JM, Lassau N, Leen E, Piscaglia F An EFSUMB introduction into Dynamic Contrast-Enhanced Ultrasound (DCE-US) for quantification of tumour perfusion. Ultraschall Med. 2012;33(4):344-351.

24. Kang M, Wang F, Liao X, Zhou P, Wang R. Intensity-modulated radiotherapy combined with endostar has similar efficacy but weaker acute adverse reactions than IMRT combined with chemotherapy in the treatment of locally advanced nasopharyngeal carcinoma. Medicine (Baltimore). 2018; 97(25):e11118.

25. Guan Y, Li A, Xiao W, et al. The efficacy and safety of Endostar combined with chemoradiotherapy for patients with advanced, locally recurrent nasopharyngeal carcinoma. Oncotarget. 2015;6(32): 33926-33934.

26. Jin T, Jiang F, Jin QF, Piao YF, Chen XZ. Endostar Combined with Gemcitabine and Cisplatin Chemotherapy for Patients with Metastatic Nasopharyngeal Carcinoma: an Update. Transl Oncol. 2018;11(2): 286-291.

27. Weidner N, Semple JP, Welch WR, Folkman J. Tumor angiogenesis and metastasis - correlation in invasive breast carcinoma. $N$ Engl J Med. 1991;324(1):1-8.

28. Hernández-Agudo E, Mondejar T, Soto-Montenegro ML, et al. Monitoring vascular normalization induced by antiangiogenic treatment with (18)F-fluoromisonidazole-PET. Mol Oncol. 2016;10(5):704-718.

29. Wegner CS, Hauge A, Simonsen TG, Gaustad JV, Andersen LMK, Rofstad EK. DCE-MRI of Sunitinib-Induced Changes in Tumor Microvasculature and Hypoxia: A Study of Pancreatic Ductal Adenocarcinoma Xenografts. Neoplasia. 2018;20(7):734-744.

30. Jiang XD, Dai P, Qiao Y, Wu J, Song DA, Li SQ. Clinical study on the recombinant human endostatin regarding improving the blood perfusion and hypoxia of non-small-cell lung cancer. Clin Transl Oncol. 2012; 14(6):437-443.

31. Leen E, Averkiou M, Arditi M, et al. Dynamic contrast enhanced ultrasound assessment of the vascular effects of novel therapeutics in early stage trials. Eur Radiol. 2012;22(7):1442-1450.

32. Wilson SR, Burns PN. Microbubble-enhanced US in body imaging: what role? Radiology. 2010;257(1):24-39.

33. Mori N, Mugikura S, Takahashi S, et al. Quantitative Analysis of ContrastEnhanced Ultrasound Imaging in Invasive Breast Cancer: A Novel Technique to Obtain Histopathologic Information of Microvessel Density. Ultrasound Med Biol. 2017;43(3):607-614.

34. Li X, Li Y, Zhu Y, Fu L, Liu P. Association between enhancement patterns and parameters of contrast-enhanced ultrasound and microvesse distribution in breast cancer. Oncol Lett. 2018;15(4):5643-5649.
OncoTargets and Therapy

\section{Publish your work in this journal}

OncoTargets and Therapy is an international, peer-reviewed, open access journal focusing on the pathological basis of all cancers, potential targets for therapy and treatment protocols employed to improve the management of cancer patients. The journal also focuses on the impact of management programs and new therapeutic agents and protocols on

\section{Dovepress}

patient perspectives such as quality of life, adherence and satisfaction. The manuscript management system is completely online and includes a very quick and fair peer-review system, which is all easy to use. Visit http://www.dovepress.com/testimonials.php to read real quotes from published authors. 